\title{
La apuesta simbólica de la alteridad en el campo literario. Una aproximación sociológica a la trayectoria artística de Najat El Hachmi
}

\author{
Francesco Bellinzis
}

Investigador independiente

f.bellinos@gmail.com

Recepción: 14-12-2020

Aceptación: 15-07-2021

Publicación: 07-10-2021

\section{Resumen}

En este artículo se analiza la trayectoria literaria de la escritora de origen inmigrante Najat El Hachmi desarrollada desde 2004 hasta 2018. Se observa la literatura como un instrumento de emancipación respecto a los vínculos dominantes — patriarcales, culturales y familiares-, a partir de las herramientas estratégicas del lenguaje y el imaginario. Por un lado, este artículo desea analizar la literatura como un posible espacio de representación para el sujeto inmigrante; por el otro, intenta comprender cuáles son las reglas de este espacio de representación. Siguiendo el enfoque bourdieusiano de la teoría del campo, se analizan las relaciones de poder que atribuyen a estos productos artísticos un valor simbólico. Este valor se relaciona con la negociación de la alteridad migrante que determina la posición de la autora en el campo. La trayectoria literaria se analizará utilizando una metodología cualitativa e interpretativa: mediante la reconstrucción de los datos biográficos, el análisis sociológico de sus novelas y la recepción de sus obras tanto en la prensa como en la crítica literaria.

Palabras clave: sociología de la literatura; migración; diversidad cultural; plurilingüismo; transnacionalismo

Abstract. The symbolic gamble of alterity in the literary field. A sociological approach to the artistic trajectory of Najat El Hachmi

This paper analyzes the literary trajectory of the migrant writer Najat El Hachmi, included between 2004 and 2018. It observes literature as a tool of emancipation against dominant bonds: patriarchal, cultural and familial. On the one side, this paper pretends to analyze literature as a space of representation for a migrant writer, on the other side, it observes the internal roles of it. From the Bourdieu's field theory approach, it is explored the relation of power that gives a symbolic value to this cultural product. This value is linked to the negotiation of an alterity that determines the writer's position in the field. The literary career will be analyzed using a qualitative and interpretative methodology with biographical data, sociological analysis of novel and its reception in Spanish press and literary critics.

Keywords: sociology of literature; migration; cultural diversity; multilingualism; transnationalism 


\section{Sumario}

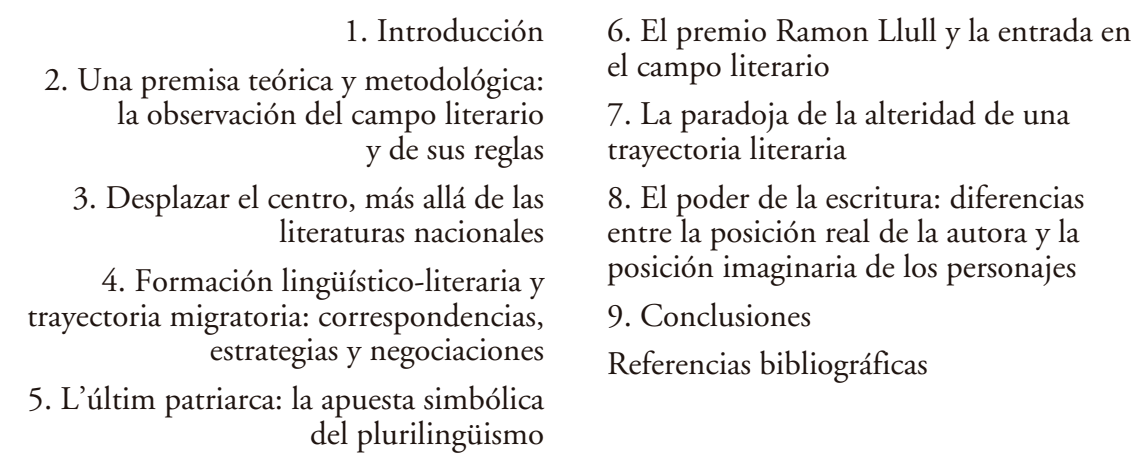

\section{Introducción}

En este artículo se analiza la posición en el campo literario de la escritora de origen marroquí Najat El Hachmi a partir de las relaciones entre su trayectoria migratoria y su trayectoria literaria. Considerando la literatura como un instrumento de representación con sus reglas, formas y discursos propios, se pretende comprender cómo una mujer de origen inmigrante puede tener dentro de este campo una voz y una forma de autorrepresentación. Antes de introducir el sujeto y el objeto de estudio específicos, se presenta y justifica el interés hacia la denominada literatura de la migración y hacia la compleja pero creativa relación entre la sociología y la literatura. La primera premisa hace referencia al desafío de las voces subalternas que por medio de la literatura han respondido al centro dominante del poder colonial e imperialista europeo (Spivak, 1990). Este espacio también ha sido alcanzado por autores inmigrantes originarios de países no occidentales (Gnisci, 2003; Mengozzi, 2013). En las últimas dos décadas, tanto en el mundo editorial como en el académico se han interesado por las voces de los autores inmigrantes. Uno de los objetivos en los estudios literarios europeos sobre las obras de estos escritores (Gnisci, 1998; Cazenave, 2003; Ricci, 2014) ha sido observar cómo la novela contrapone al discurso que se hace sobre el sujeto-otro y subalterno la voz de la alteridad, que se autorrepresenta como una voz casi siempre negada y silenciada.

Si la primera premisa necesaria para introducir esta investigación empieza por desvelar la importancia de la literatura para dar voz al sujeto inmigrante, la segunda premisa trata de comprender cuáles son las reglas de este espacio de representación. Para entender cómo el otro representado se convierte en el otro que se autorrepresenta, es fundamental conocer sociológicamente las relaciones simbólicas que el inmigrante aceptado como otro, publicado como alteridad y constituido como extraño establece con el campo específico de la literatura. A partir de esta inquietud, surgen las siguientes preguntas: ¿cuáles son las correspondencias entre la trayectoria migratoria de un autor/a y su trayectoria 
literaria?, ¿qué papel tiene la negociación de la alteridad en una estrategia de posicionamiento, visibilidad y consagración en el campo literario?

Con este artículo, se pretende analizar la trayectoria literaria de la escritora catalana de origen marroquí Najat El Hachmi, en el periodo comprendido desde 2004 hasta 2018. El análisis tendrá en cuenta un factor clave: las reglas de un mundo particular que Bourdieu (1995: 65) define como campo literario. Siguiendo la propuesta teórica del sociólogo francés, el presente análisis introduce en el debate sobre la relación con el otro migrante las relaciones simbólicas producidas en el contexto de la literatura contemporánea a partir de la comprensión de sus reglas internas. Si la sociología se ha interesado desde siempre por la constitución simbólica del otro, como extraño (Bauman, 1995), extranjero (Simmel, 1977) o inmigrante (Sayad, 2014), se considera todavía muy escaso y marginal el estudio sociológico de la alteridad migrante en el campo literario. Con esta investigación, no se pretende explicar un fenómeno con descripciones y relaciones entre variables, sino con una comprensión e interpretación del mismo. En la tabla 1 se describen los objetivos y las preguntas de la investigación. El artículo se divide en nueve secciones: la presente introducción, siete apartados, más las conclusiones. En el primer apartado, se integran los aspectos que se refieren a las decisiones de tipo teórico y metodológico. En el segundo, se revisan las nuevas aportaciones a la teoría del campo literario a partir de un enfoque transnacional. A partir del tercer apartado, se incluye la primera parte empírica que analiza la producción literaria de El Hachmi. El cuarto pone énfasis en la novela más representativa, y el quinto analiza cómo a través de esta obra la autora accede al campo literario. El sexto apartado expondrá las relaciones simbólicas entre la trayectoria literaria y la trayectoria migratoria. El séptimo, finalmente, se encarga de resaltar las diferencias entre la posición real de la autora y la posición imaginaria de los personajes de sus novelas.

Tabla 1. Objetivos y preguntas de investigación

\begin{tabular}{lc}
\hline Objetivos & Preguntas de investigación \\
\hline $\begin{array}{l}\text { 1. Comprender cómo la literatura permite } \\
\text { a una mujer de origen inmigrante tener } \\
\text { un espacio, una voz, una forma de }\end{array}$ & $\begin{array}{l}\text { 1. ¿En qué medida la novela es un } \\
\text { instrumento de representación para una }\end{array}$ \\
$\begin{array}{l}\text { autorrepresentación. } \\
\text { 2. Analizar las correspondencias entre la de origen inmigrante? } \\
\text { estrategia de posicionamiento de la autora } \\
\text { y la negociación del origen inmigrante. }\end{array}$ & $\begin{array}{l}\text { 2. ¿Cómo negocia la escritora su origen } \\
\text { en el campo literario? la relación entre el proceso de }\end{array}$ \\
$\begin{array}{l}\text { 3. Descubrir si estas novelas son el síntoma } \\
\text { de relaciones trasnacionales y cómo deter- } \\
\text { minan la posición de este sujeto de origento en un campo local y las } \\
\text { inmigrante en un contexto local. }\end{array}$ & $\begin{array}{l}\text { relaciones trasnacionales que han orientado } \\
\text { la producción literaria de la autora? }\end{array}$ \\
$\begin{array}{l}\text { 4. Investigar las relaciones entre trayectoria } \\
\text { artística y trayectoria migratoria. }\end{array}$ & trayectoria migratoria de esta novelista \\
\end{tabular}

Fuente: elaboración propia 


\section{Una premisa teórica y metodológica: la observación del campo literario y de sus reglas}

Empezar un estudio sobre el complejo diálogo entre literatura y sociedad significa subrayar desde el principio el peligro de banalizar un texto literario como reflejo de una ideología y el peligro de transformar los personajes de una novela en marionetas teóricas desarraigadas de su mundo específico (Turnaturi, 2003). La compleja relación propuesta en términos deterministas por los primeros enfoques sociológicos de la literatura ha puesto en peligro una disciplina que, como afirma Sapiro (2014: 9), era en su origen demasiado literaria para los sociólogos o demasiado sociológica para los críticos de la literatura. Sapiro (2014) relaciona los autores de esta fase presociológica con la teoría del reflejo. Tanto las posiciones de Lukács (1966) como las de Goldmann (1967) podemos reconducirlas a esta teoría mediante la observación de la novela como reflejo de la realidad social.

El sociólogo que pone en evidencia los límites de esta posición es Pierre Bourdieu, quien se percata de cómo en este enfoque se ignora por completo «el efecto de refracción que ejerce el campo de producción cultural» (Bourdieu, 1995: 334). Con el enfoque bourdieusiano, podemos proceder a un análisis que explique las prácticas culturales de esta escritora ante la relación de una realidad social específica: el campo literario. El espacio estructurado se constituye a partir de relaciones de fuerza entre agentes (críticos, editores, traductores, escritores) e instituciones (editoriales, mundo académico). Este elemento innovador respecto al enfoque marxista permite estudiar la literatura a partir de sus propias reglas, y los escritores a partir de un capital cultural específico (Bourdieu, 1993; Boschetti, 2003). El capital cultural es una acumulación de una serie de habilidades y conocimientos adquiridos por medio de la familia, la escuela o una determinada experiencia social (Bourdieu, 1979). El mismo autor (1988) define la acumulación de estas competencias relacionadas con el lenguaje como capital lingüístico. Casanova (2001) define el capital relacionado con un idioma literario como "capital literario», que es la acumulación determinada por los mecanismos de legitimación internos al campo literario.

Con las descripciones de campo y de capital cultural expuestas hasta ahora, podemos dibujar algunos de los funcionamientos internos de este microcosmos. Por lo tanto, sería oportuno comprender cómo el escritor se mueve en este terreno. Podemos resumir la teoría de Bourdieu con esta fórmula: (habituscapital) + campo $=$ prácticas. Un escritor, a partir de su habitus y un capital específico, se posiciona en el campo, y es posible desde esta posición explicar la dinámica de sus prácticas. El habitus, según Bourdieu (1995), es lo social incorporado que orienta las conductas. Podemos explicar las representaciones literarias de un autor, su toma de posiciones y su gusto estético no desde la base de una obediencia a reglas preestablecidas, sino de una orientación socialmente determinada.

La fórmula bourdieusiana antes mencionada orienta el enfoque teórico del presente artículo hacia un escepticismo crítico necesario para comprender 
las relaciones de fuerza que han determinado la trayectoria artística de Najat El Hachmi. Uno de los aspectos más importantes de esta teoría es que se aleja de «los partidarios de la espontaneidad creadora» (Bourdieu, 1995: 118) para investigar qué reglas ofrecen al autor un derecho de entrada al campo. Esta posición crítica relacionada con la teoría del campo se ha utilizado con el primer objetivo de este artículo: comprender las reglas del universo literario analizándolo como un posible espacio de autorrepresentación para el sujeto inmigrante. Los intereses que motivan esta investigación están enfocados hacia una autora en concreto, una mujer de origen inmigrante. Los estudios más relevantes de la sociología de la literatura se han enfrentado desde siempre a un desafío metodológico: el número de escritores analizados considerando el campo literario como un objeto de estudio que dificulta las generalizaciones. Bourdieu (1995), por ejemplo, enfoca su comprensión del campo literario francés en Flaubert y una novela específica, La educación sentimental. El sociólogo justifica esta elección a partir de un enfoque sociohistórico de la novela en relación con la conquista de la autonomía artística. Un análisis sobre la posición de Najat El Hachmi en el campo literario se justifica al considerarla como una de las primeras novelistas de origen inmigrante procedentes de un país no occidental que aporta cualidades externas fruto de la misma experiencia migratoria en la literatura catalana.

El primer acercamiento a este objeto de estudio hace referencia a una metodología sociológica de la reconstrucción de los datos biográficos (Bourdieu, 1995; Lahire, 2011). Estos datos no se relacionan de forma mecanicista con la producción de la novelista, por el contrario, todos los condicionamientos sociales que se analizan se enlazan al concepto de habitus bourdieusiano. Si por un lado Bourdieu analiza (ibidem) cómo una determinada condición económica y social orienta el acceso al campo de un escritor, por otro, Lahire (2011) introduce un elemento fundamental en este enfoque sociológico: la acción de la escritura sobre el escritor. La acción social de la escritura y la entrada en el campo pueden determinar, según Lahire (2011), una experiencia socializadora. Con esta separación, podemos analizar las obras de Najat El Hachmi no solo como el fruto de una experiencia migratoria, sino también ver qué efecto han tenido la escritura y su ingreso en el campo literario en su propia condición de inmigrante. Este aspecto permite reflexionar sobre dos conceptos clave de la sociología de la literatura: la posición y la trayectoria literaria. Podemos considerar la posición, según Bourdieu (1995), como el espacio que ocupa el escritor en el campo literario, determinado por su habitus y las posibilidades de posicionamiento que ofrece el mismo, a partir de la puesta en juego de unos capitales culturales. La trayectoria, por otro lado, se puede reconducir a las prácticas literarias desarrolladas en el tiempo a partir de una posición específica. Aunque la posición se relaciona con el espacio y la trayectoria con el tiempo, las dos están interconectadas: la posición condiciona el desarrollo de una trayectoria artística y, a su vez, una determinada trayectoria puede modificar la posición de un autor. Las aportaciones de Lahire (2011) se refieren a esta posibilidad, que supera la mirada hacia una posición estática y casi inmutable en el enfoque 
bourdieusiano. En este artículo, se plantea el habitus a partir de una dimensión más plural dentro del contexto de las migraciones, donde las orientaciones son transnacionales y no están enraizadas a una sola condición, sino a múltiples condiciones. Este planteamiento está enlazado al segundo y cuarto objetivo de este estudio: la observación e interpretación de la negociación estratégica de la experiencia migratoria, y la relación entre trayectoria migratoria y trayectoria literaria.

Para esta investigación se ha utilizado una metodología cualitativa e interpretativa (Bryman, 2012). A diferencia de otros estudios fundamentales de la sociología de la literatura como el estudio de Bourdieu (1995) sobre Flaubert o de Lahire (2010) sobre Kafka, en este análisis se introduce una entrevista semiestructurada a El Hachmi como material complementario. ${ }^{1}$ Otros materiales son las novelas, entrevistas a la autora en la prensa, la recepción de sus obras en la crítica literaria y en la prensa (La Vanguardia, El País, El Periódico). $\mathrm{El}$ análisis de la trayectoria literaria comprende el análisis sociológico de su primer libro autobiográfico, Jo també sóc catalana (2004), y de las novelas de su trilogía, L'últim patriarca (2008), La filla estrangera (2015) y Mare de llet $i$ mel (2018). No se toma en consideración La caçadora de cossos (2011) por ser la única novela que no está conectada con la experiencia migratoria. El análisis de esta trayectoria artística se enlaza con la observación y la lectura más profunda de su obra más representativa, L’últim patriarca, premio Ramon Llull de las letras catalanas en 2008. Siguiendo la metodología de Bourdieu (1995: 19), se puede considerar como análisis sociológico de la novela «una lectura estrictamente interna», que saca a la luz la configuración del espacio social imaginado donde se mueven los personajes y su relación no mecanicista con la estructura del espacio social donde se mueve el autor. En este caso concreto, se tratará la obra como un síntoma de condiciones sociales, en particular su origen migrante, observando e interpretando cómo estas se han refractado (no reflejado) en el prisma del campo literario, objetivadas a partir de sus mecanismos y en relación con un capital cultural específico: el lenguaje literario (Casanova, 2001). Además de este aspecto, se ha introducido en la caja de herramientas teóricas y metodológicas bourdieusianas un enfoque transnacional. En este artículo se superan las fronteras nacionales que delimitan la unidad de análisis. Posteriormente, se introducen las herramientas teóricas del transnacionalismo literario, que se enlazan al tercer objetivo del estudio y que son necesarias para el análisis de estas novelas en las que entran en contacto diferentes herencias culturales, imaginarios, representaciones y lenguas.

\section{Desplazar el centro, más allá de las literaturas nacionales}

Como afirma Steve Vertovec (2004: 9), el transnacionalismo hoy parece estar en cualquier contexto académico, debido a que en los diferentes enfoques que

1. La entrevista semiestructurada a la autora se enfoca en el desarrollo de su trayectoria literaria y en su experiencia migratoria. 
cruzan estas disciplinas existe una tendencia a ocuparse, entre otros asuntos, de las migraciones y las relaciones sociales que cruzan las fronteras nacionales. Este es un aspecto fundamental del enfoque transnacional es su posición crítica hacia el nacionalismo metodológico. Recurriendo a la descripción de Esperanza Bielsa (2016: 2), podemos acercarnos al significado de este término y a su comprensión sociológica. Bielsa (ibidem) explica cómo, según el nacionalismo metodológico, el estado nación es el centro natural de la sociedad moderna, y por lo tanto se ignoran las relaciones transnacionales y las múltiples identidades que se establecen más allá de la nación. En este enfoque residen no solo los problemas teóricos y metodológicos de las ciencias sociales para explicar un fenómeno tan complejo como las migraciones contemporáneas, sino también, como afirman Wimmer y Glick Shiller (2002: 67), la tendencia a describir a los inmigrantes como un riesgo para la seguridad nacional, así como una alteración cultural. Los enfoques transnacionales han puesto a la luz la necesidad de estudiar y observar las migraciones de formas diferentes. La necesidad de este enfoque es la de considerar las relaciones del sujeto inmigrante no exclusivamente tomando como punto de partida el estado nación, sino por medio de sus relaciones entre fronteras.

El enfoque transnacional ha tenido también un papel determinante en los estudios literarios. Los intereses por los intercambios transnacionales de los bienes simbólicos determinaron un pasaje importante en la sociología de la literatura, que extendió su mirada desde el contexto nacional a uno mundial (Sapiro, 2013). A finales de los setenta, emergen nuevos intereses por la que será definida como world literature, que incluye también a los autores poscoloniales, de minorías étnicas o inmigrantes. Se consideran parte de la literatura mundial todas aquellas obras literarias que circulan, tanto traducidas como en lengua original, fuera de su contexto de producción original, cruzando las fronteras nacionales y transnacionales de su cultura de origen (Damrosch, 2003; Bielsa, 2016). Para entender el concepto de world literature, es necesario hacer referencia a la idea goethiana de Weltliteratur que supera el concepto de literatura nacional e introduce el concepto de literatura mundial. Como sostiene Moretti (1996: 37), en el límite del ideal goethiano (Weltliteratur) se puede reconducir al «universalismo del centro». El crítico literario italiano se pregunta si este ideal se refiere a la literatura de la humanidad o más bien a la literatura del imperialismo. Sin duda, en el ideal goethiano faltaba aquella conciencia sobre las relaciones de fuerza y poder entre las diferentes lenguas literarias que serán objeto de los estudios poscoloniales (Said, 1979; Spivak, 1990).

Gracias al avance de los estudios poscoloniales, se contribuyó a alimentar el interés no solo por la producción artística de escritores procedentes de las excolonias, sino también la edición de escritores de origen inmigrante en el debate y la crítica literaria contemporánea. En los textos históricos y literarios occidentales, no podemos encontrar, como considera Spivak (1990), la verdadera subjetividad de los dominados. En este sentido, el intelectual palestino Said, en el texto Beginnings (1975), reflexiona sobre la figura del intelectual inmigrante o exiliado. En otra obra fundamental, Orientalism (1979), Said 
investigó a partir del análisis del discurso la manera en que Occidente construye la imagen de un sujeto otro y subalterno. El otro orientalizado es construido sobre la base de categorías inmutables que refuerzan los prejuicios. Este tipo de análisis del discurso permite recontextualizar las obras del canon occidental.

La literatura tiene una enorme importancia en un proceso de colonización o de descolonización, sin embargo, como sostiene Boschetti (2012), los estudios poscoloniales, aunque son un instrumento teórico útil para repensar y redefinir la crítica literaria eurocéntrica, no ofrecen una solución metodológica adecuada para un análisis con enfoque sociológico. El problema tanto teórico como metodológico de los estudios poscoloniales es que excluyen del análisis a los agentes que crean y recrean las relaciones de fuerza en el campo literario, como los editores, traductores, académicos, lectores o la prensa. En este sentido, la teoría de Bourdieu, que se incorpora en las aportaciones de Casanova (2001) y Sapiro (2009), ofrece unas herramientas teóricas y metodológicas útiles para estudiar la obra de una escritora inmigrante sin ignorar todos los agentes que participan y determinan su posición en el campo. Casanova (2001) examina las relaciones de fuerza entre diferentes literaturas nacionales en un análisis transnacional del campo literario, sin ignorar que los escritores, al moverse entre diferentes lenguas y literaturas nacionales, deben adherirse a las «leyes específicas y a las fuerzas inscritas en la estructura desigual del universo literario, (...) conscientes de que deben ser consagrados en esos centros de poder para obtener alguna oportunidad de sobrevivir» (Casanova, 2001: 65). Por otro lado, Sapiro (2009) reconduce esta mirada como parte de un análisis de la literatura vista como un campo con su lucha interna, donde en el caso del campo transnacional los dominados ocupan una posición marginal para escribir con los recursos de un país que está excluido en los intercambios de un mercado global. En esta dirección, Bruillette (2007) analiza cómo en el campo literario transnacional se produjo una mercantilización de lo exótico con reglas específicas dentro de un mercado editorial globalizado, donde los escritores poscoloniales pueden negociar la propia alteridad como elemento de originalidad y de valor añadido. Esta mercantilización es alimentada por una ambivalencia entre el lugar de recepción de la novela poscolonial (el norte global) y los sujetos que están representados en estos textos (el sur global).

Estas últimas perspectivas mencionadas guían el marco teórico transnacional de este estudio, sin renunciar a la teoría del campo. Como se ha analizado en este apartado, estos avances teóricos han aportado herramientas metodológicas para analizar la literatura como universo social más allá del estado nación como unidad de análisis. De todas formas, respecto a las relaciones de fuerza entre literaturas periféricas y centrales, dominantes y dominadas, que analizan en sus estudios tanto Casanova (2001), Sapiro (2009) como Bruillette (2007), en este artículo se analiza a una escritora de origen inmigrante que se posiciona dentro de un campo nacional aportando cualidades y características externas. Un análisis de las obras de Najat El Hachmi introduce en el debate del transnacionalismo literario la observación de las relaciones transnacionales, poscoloniales y de poder, dentro de una misma literatura local, a 
través de una mujer que creció en esta sociedad aportando a su campo local cualidades externas desde la contracultura de la marginalidad inmigrante. En las próximas secciones se analizará en detalle este aspecto.

\section{Formación lingüístico-literaria y trayectoria migratoria: correspondencias, estrategias y negociaciones}

Najat El Hachmi nació en Beni Sidel (Marruecos), en 1979, en el seno de una familia de clase trabajadora, pero se mudó a la pequeña ciudad catalana de Vic a la edad de ocho años por medio de un proceso de reagrupación familiar. Su formación escolar se realizó en Cataluña, donde aprendió a leer y escribir. Por lo tanto, El Hachmi es una inmigrante de la generación 1.5. En sociología de las migraciones se utiliza esta expresión para definir «a los hijos que los mismos inmigrantes han traído a España antes de que pudieran iniciar su socialización escolar» (Aparicio y Tornos, 2006: 24). La escritora nació en Marruecos y, por lo tanto, técnicamente no es una inmigrante de segunda generación, porque no nació en la sociedad de acogida, de todas formas, como subrayan Aparicio y Tornos (ibidem), este grupo "comparte una misma sensibilidad generacional con la estrictamente llamada segunda generación».

La escritora se escolarizó en la lengua del país de adopción y sustituyó la lengua materna en los procesos de aprendizaje, aspecto que analiza en su primera obra (El Hachmi, 2004). El primer libro de El Hachmi, Jo també sóc catalana (2004), es de corte autobiográfico y reflexiona sobre la sensibilidad generacional de los hijos de los inmigrantes. Es importante evidenciar en este análisis cómo Najat El Hachmi, a partir del bajo capital económico y cultural de su familia, elije la lengua catalana como elemento estratégico de su trayectoria artística. Aunque el catalán tiene mayor peso en su formación literaria, también el tamazight es reivindicado por ella como un valor todavía no consagrado. La novelista hace esta afirmación con relación a la cultura oral amazigh, como un conocimiento heredado: «És més aviat un do de la meva mare. La meva mare és analfabeta, però té una capacitat única per explicar històries» (El Hachmi en una entrevista de Puigtobella a Barcelona Metròpolis, «La ciutat multilingüe», 2016: 6). Su primera obra de corte autobiográfico (1995) compara el tamazight, de minoría lingüística en Marruecos, con el catalán, de igual limitación en España: «La teva altra llengua materna, el català, fou en altres temps perseguida i menystinguda, no en va la teva mare les sent com dues llengües germanes» (El Hachmi, 1996: 84). La relación que hace de las dos lenguas cruza nuevas fronteras identitarias (Ingenschay, 2011).

Ser hija de inmigrantes puede orientar la manera de ver el espacio social y la forma de reproducirlo en una obra literaria. Este condicionamiento está conectado, según la formula sociológica de Bourdieu (1993), con el campo literario y con las posibilidades que ofrece. Por esta razón, entra en juego la dimensión histórica del contexto literario local y sus relaciones transnacionales con otros contextos. Respecto al ámbito local donde se sitúa la escritora, podemos mencionar una consideración del traductor Josep Marco (2010: 83), en relación 
con la literatura catalana como lengua interrumpida. El traductor subraya la necesidad de una nueva acumulación de capital literario, no porque la literatura catalana sea una literatura menor o por no tener referencias antiguas y una importante comunidad geográfica, sino por razones históricas, pues se trata de una tradición literaria interrumpida a causa de la censura franquista. La escritora entra en el campo en un momento peculiar de la literatura catalana, cuando se intenta superar, gracias también a un apoyo político y económico determinante, una posición periférica y marginal. La escritora se formó en la Cataluña posfranquista, cuando el catalán como capital literario reconquistó otro peso en el campo cultural. La literatura catalana, aunque ha recuperado su importancia en el contexto local, se enfrenta a todas las exigencias de un mundo editorial globalizado en torno a una realidad literaria mundial, donde los localismos son obligados a ser traducidos en los procesos de circulación transnacional de los bienes simbólicos (Benvenuti y Ceserani, 2012; Casanova, 2001; Boschetti, 2012). Muchas literaturas nacionales han aprovechado a los escritores poscoloniales o inmigrantes con el fin de acumular un capital literario necesario para un reposicionamiento en el espacio internacional (Casanova, 2001; Gnisci, 2003).

Dicho proceso hubiera sido probablemente diferente si El Hachmi hubiese elegido el castellano como lengua literaria, ya que la negociación de una contaminación cultural la hubiese posicionado en la sombra respecto a la magnitud de las contaminaciones literarias del boom latinoamericano. Este aspecto lo sufrieron los autores marroquíes en lengua castellana, que se quedaron en una posición totalmente periférica respecto al proceso de transculturación literaria de los autores latinoamericanos (Rama; 1985; Ricci, 2014). Por otro lado, cuando El Hachmi publica su primer libro, en 2004, la presencia de autores extranjeros de países no occidentales en lengua catalana es casi nula. De todas formas, es importante mencionar que en aquel mismo año otra escritora de origen amazigh, Laila Karrouch (2004), publica su primer libro, De Nador a Vic. El filólogo Ricci (2014: 217) posiciona tanto a Karrouch como a El Hachmi en una misma área literaria de escritoras imazighen catalanas. El Hachmi y Karrouch entran en el campo literario local compitiendo con un número muy exiguo de colegas con la misma trayectoria, por lo tanto, tienen que luchar para que la diversidad cultural se acepte en este contexto, pero no compiten con otros escritores migrantes. En este caso, es importante subrayar que El Hachmi, a diferencia de Karrouch, tardó solo pocos años, después de su primera obra, en pasar de una experiencia vocacional a una experiencia profesional como novelista (Heinch, 2000). En los próximos apartados, se analizará en detalle este último aspecto y se considerará la trayectoria de El Hachmi como un objeto de estudio estratégico para la comprensión de las relaciones entre trayectoria migratoria y trayectoria literaria.

\section{L'últim patriarca: la apuesta simbólica del plurilingüismo}

Después de su obra autobiográfica, Jo tambe sóc catalana (2004), en 2008 El Hachmi publica L'últim patriarca. Esta obra es una novela que cuenta la 
historia de la saga familiar de los Driuch, que se divide en tres generaciones. La primera generación de los Driuch representa a los abuelos de una familia amazigh del norte de Marruecos. La segunda es personificada por el patriarca Mimoun y su mujer, y la tercera por su hija, la voz narrativa. Mimoun emigra a Cataluña dejando atrás el pueblo rifeño y una sociedad rural. En esta parte de la obra la experiencia migratoria condiciona y mueve a todos los personajes dentro de un espacio social donde se subrayan sus marginalidades. Mimoun encarna el individuo inmigrante que induce el primer avenimiento narrativo: la emigración y la trasformación de la familia Driuch. Es necesario, por lo tanto, hacer un análisis que conecte el contexto histórico y social de la inmigración marroquí en Cataluña con el punto de vista singular de la narradora, hija de inmigrantes. Si Mimoun encarna al inmigrante con bajo capital económico y cultural que emigró en los años ochenta para suplir la necesidad de mano de obra barata en Cataluña, la representación, por parte de su hija, encarna la imagen de los hijos de los inmigrantes respecto a los padres. El lector, que sigue el viaje de la narradora desde el norte de Marruecos hacia Cataluña, desconoce a la protagonista en toda la primera parte, y es esta invisibilidad la que ofrece todavía más poder a una narración que intenta entrar en los aspectos más prohibidos y ocultos para la mirada de una joven en una comunidad con fuertes raigambres en el islam (Ricci, 2010). Este conflicto generacional es el segundo avenimiento narrativo: la trasformación de la familia Driuch como parte de la condena del patriarcado.

El Hachmi utiliza el catalán como capital lingüístico y literario y lo relaciona con otros idiomas como el árabe y el tamazight. En esta novela las dos culturas se enfrentan y se traducen, tal y como afirma Vidal Claromonte (2012: 249): «Las identidades que aparecen en L'últim patriarca son sujetos que desde luego no son puros, sino que están en permanente traducción porque, como nos recuerda Cronin, la condición del migrante es la de un ser traducido». El movimiento entre un mundo y otro, que Vidal Claromonte explica a lo largo de la metáfora de Cronin (1998) del ser traducido, no está exento de relaciones de poder. Estas relaciones se pueden explicar en dos momentos distintos: la posición de la lengua con el polo legítimo en el capo del poder y su desterritorialización. Najat El Hachmi obtiene un espacio de representación que, en su cultura de origen, una mujer amazigh con su clase social no hubiera podido alcanzar. Su lejanía respecto al lenguaje legítimo está presente tanto en relación con la sociedad de origen como con la sociedad de acogida. Cuando Bourdieu (1988) analiza el mercado lingüístico y el poder simbólico de la lengua, hace una distinción fundamental entre el campo de poder y el campo literario. En el campo literario, el proceso de consagración de una lengua y su forma de utilizarla no corresponden siempre al lenguaje legítimo del poder político. Por ejemplo, Bourdieu (1988: 59) menciona a Victor Hugo y su consciente oposición al lenguaje legítimo del poder político mediante una mímesis del lenguaje del pueblo. Con esta novela, la autora se caracteriza por esta contradicción, porque se posiciona en el punto más lejano del lenguaje legítimo y dominante por no tener ni el derecho ni las herencias culturales para 
acercarse a él. Paradójicamente, el lenguaje ilegítimo, devaluado y estigmatizado que utiliza una mujer de origen inmigrante de clase trabajadora ha podido, debido a los mecanismos únicos y peculiares del campo literario, convertirse en un valor negociable.

El juego que construye El Hachmi con su plurilingüismo consiste en utilizar el catalán en un lugar donde esta lengua es desconocida (Marruecos) y hace lo mismo con el tamzight, desterritorializándolo en el contexto europeo (Bueno Alonso, 2010). Al situar el tamazight fuera del territorio, la autora negocia la propia alteridad en el campo literario catalán proponiendo la idea de que este necesita ser contaminado para sobrevivir como lengua literaria. En este caso, es la contaminación de una joven escritora de la clase obrera migrante la que nos ubica en el polo más lejano del centro dominante. En relación con esto, Najat El Hachmi comenta en la entrevista realizada por Ernest Alós en El Periódico (1 de febrero de 2008): "O la literatura catalana se deja contaminar o se muere». Con estas observaciones, se introducen los primeros elementos del análisis, necesarios para contestar a la segunda pregunta de la investigación en referencia a la negociación del origen inmigrante. Estos elementos se analizarán detalladamente más adelante.

Con su primera novela, Najat El Hachmi no solo defiende la intromisión de otras lenguas en la literatura catalana, como el tamazight y el árabe, sino que considera necesario este proceso de contaminación para la supervivencia del catalán en el mundo literario. Este desafío comienza con la ruptura del vínculo orgánico entre lengua y el estado nación, que defiende el paradigma monolingüe, lo que presupone una relación directa, biológica y exclusiva entre el sujeto y su lengua materna. El plurilingüismo en literatura, según Yildiz (2012), tiene una función subversiva: la de librarse y desafiar el concepto esencialista de lengua materna asociado al paradigma monolingüe. En esta novela, el plurilingüismo tiene también una connotación de género. La narradora utiliza una lengua y una forma narrativa desconocida en su contexto de origen para profanar los vínculos patriarcales de esta cultura (Bellinzis, 2019). En este caso, es necesario recordar una observación del intelectual argelino Lamri (2002: 2), que consideraba la lengua extranjera estratégicamente funcional para un proceso de liberación y profanación. La estrategia de la novelista es volver a su cultura de origen con una lengua y una forma narrativa allí desconocida, es decir, que utiliza el catalán literario y la novela para espiar en lugares prohibidos.

El análisis de la estrategia analizada durante esta sesión ha intentado ir más allá de la relación dicotómica mujer-novela para comprender esta complejidad por medio de la puesta en juego de un capital lingüístico, en este caso concreto relacionado con el plurilingüismo. El habitus lingüístico de la autora choca con las reglas del sistema político dominante, esto es, con el aprecio desmesurado de lo nacional y con el desprecio de lo ajeno. Como afirma Bourdieu (1988), cada discurso es el resultado entre un habitus lingüístico y un sistema de reglas. Con base en su habitus lingüístico, El Hachmi necesita subvertir y cambiar el sistema de reglas a través de una forma de expresarse marcada culturalmente, que reivindica como elemento de legitimación. Los vínculos que producen sus 
prácticas lingüístico-literarias se pueden reconducir con una mirada de orientaciones dobles o múltiples, bien sea del lugar de origen al lugar de llegada, o en el contexto de la literatura mundial. Esto lleva a repensar la estructura estructurada que es el habitus dentro del contexto trasnacional de las migraciones (Guarnizo, 1997).

\section{El premio Ramon Llull y la entrada en el campo literario}

Partiendo del análisis sociológico de la novela, se ha observado cómo la escritora ha conectado una realidad local con una realidad de la migración trasnacional. La entrada de la autora en el campo está relacionada con dos referencias culturales periféricas: la comunidad rural del Rif y una pequeña ciudad de provincia. En esta entrevista, la misma autora reflexiona sobre esta doble dimensión periférica en relación con su trayectoria migratoria:

El primer sitio donde aterrizas te define, te condiciona igual que el lugar donde naces. Incluso los inmigrantes piensan que uno emigra de un país a otro, de Marruecos a España, esta era la idea que teníamos allí antes de emigrar, y luego resulta que te llevan a un lugar pequeñito muy concreto, a un pueblo pequeñito muy concreto, y me preguntaba: ¿esto es España?

El problema (de esta comunidad pequeña) es que estableces círculos de relaciones con la comunidad de tu lugar de origen y con la sociedad de acogida, y depende de en que situaciones puede ser muy limitado, te da seguridad porque es un lugar muy concreto, donde tienes una red de apoyo, pero te condiciona porque no deja de ser una comunidad pequeña trasladada a un lugar pequeño y vives con un doble control. (Najat El Hachmi, en la entrevista realizada para esta investigación)

De acuerdo con Sayad (2010), no hay una inmigración, sino que hay también una emigración, como si fueran dos realidades inseparables. Sandra Gil Araujo (2010) considera a Sayad como un anticipador de los estudios transnacionales por la importancia de la inmigración global en su trabajo entre el país de origen y el receptor. Sayad estudia en su trabajo La doble ausencia (2010) la sociedad de la emigración, y utiliza la imagen de la doble ausencia para denunciar la doble cara de las migraciones que caracteriza la vida del inmigrado, es decir, lo que afecta al emigrado en las dos sociedades, la de acogida y la de origen. Si consideramos la figura de El Hachmi como la de una escritora que se relaciona con un capital cultural manteniendo como patrimonio no solo sus tradiciones culturales sino también la misma experiencia de la inmigración, podemos entonces reflexionar sobre este aspecto mediante esta pregunta: ¿a través de la escritura la autora supera la condición del doble ausente?

Esta pregunta es clave para separar dos enfoques sociológicos que pueden dialogar, pero no confundirse. Por lo tanto, no hay que olvidar que estamos analizando a una escritora inmigrante que se posiciona dentro de un campo específico como el literario. El valor de la escritura de vanguardia que propone El Hachmi pretende transgredir el campo literario local y profanar 
vínculos patriarcales de su cultura de origen, y puede ser consagrado precisamente tomando en consideración un reconocimiento por parte de los agentes dominantes del campo: prensa, editores, académicos, críticos. Si la escritura ayuda a desarrollar una identidad fluida que se mueve entre dos culturas, únicamente la cultura dominante permite a esta escritora circular y ser consagrada en el proceso que monopoliza el capital simbólico. Esta reflexión nos ayuda a contestar a la primera pregunta de la investigación enlazando la observación de este espacio de representación con los mecanismos internos al campo. Por esta razón, cabe subrayar cómo la literatura puede convertirse en un canal de superación de una condición marginal y ausente, pero solo y exclusivamente en función de sus reglas.

Un aspecto de este proceso es el éxito literario. Es necesario comprender cómo partiendo del éxito literario la escritora decide trasladarse de una realidad provincial a Barcelona para dejar de ser etiquetada como inmigrante. La autora afirma en la entrevista (realizada para este trabajo) que alejarse de una comunidad pequeña (Vic) la ayudó a superar las condiciones englobantes de su origen inmigrante. La primera etapa que se observó fue el proceso de reagrupación familiar, donde la escritora, con tan solo ocho años, se traslada de Nador a Vic. Esta experiencia determinó inevitablemente su habitus, el de una mujer inmigrante de la generación 1.5 que se escolarizó en el entorno escolar catalán. Además, fue necesario entender cómo a consecuencia del éxito literario decide trasladarse de esta realidad pequeña a Barcelona, con la voluntad de seguir siendo una escritora que hable de inmigración, para lo cual necesitó distanciarse de los controles de su comunidad de origen en Vic.

Una fase determinante de este proceso fue el otorgamiento del premio Ramon Llull, recibido en 2008 por L'últim patriarca, publicado por la editorial Planeta. Respecto al campo literario catalán y español, la editorial Planeta ocupa una posición central, así como el premio Ramon Llull es el máximo reconocimiento para la literatura catalana. Así pues, se puede afirmar que El Hachmi entra en el campo literario con L'últim patriarca. En la entrevista que se menciona abajo, la autora define la experiencia del éxito como extraña, pero es a partir de aquí que puede convertirse en escritora reconocida y, por lo tanto, tener como única ocupación el oficio de escribir:

(El éxito) es una experiencia extraña. Por un lado, es una experiencia gratificante, llegar a muchísima gente te permite dedicar la mayor parte del tiempo a escribir, es importantísimo porque la mayoría de escritores tienen otros trabajos. Los premios sirven para hacer lo que dijo una vez un escritor como Moncada: «Sirven para comprar tiempo». Es muy arriesgado, no tiene ninguna perspectiva en sí, de momento sigo en este riesgo, este riesgo lo asumí en 2008, con el primer premio. (Entrevista realizada para esta investigación)

Después del éxito literario obtenido gracias a la novela analizada, la autora se enfrenta a un ambiguo proceso de negociación donde se aleja de su comunidad de origen y de la comunidad del lugar donde creció (Vic), en consecuencia intenta dejar de ser inmigrante para convertirse en la referente local 
de la migración en el campo literario. La escritora pasa de una socialización familiar, cultural y escolar que modela un determinado habitus a una socialización secundaria que empieza con el éxito literario y la entrada en el campo. Con relación a este aspecto concreto, cabe mencionar la aportación de Lahire (2011) y la relevancia que da este sociólogo a la función social de la escritura. Las indicaciones de Lahire (ibidem) son clave para separar los datos que podemos obtener sobre el antes y el después de la entrada al mundo literario. Por esta razón, se considera que su obra L'últim patriarca, por un lado, le ha permitido entrar en el campo literario y, por otro, ha condicionado su relación, percepción y negociación del origen inmigrante. En este caso, su identificación con el colectivo inmigrante se debería relacionar con el concepto de identidad literaria. La identidad literaria, como afirma el sociólogo de la literatura Meizoz (2010), no solo está constituida por la vida personal del escritor, sino por el horizonte de recepción. Por este motivo, respecto a la posición en el campo de esta autora, se crea una peculiar paradoja, porque, aunque ella no quisiera ser etiquetada como inmigrante, está consagrada y valorizada también por esto. La alteridad y lo extraño de su escritura es parte de su éxito. En el próximo apartado se analiza en detalle este aspecto.

\section{La paradoja de la alteridad de una trayectoria literaria}

A consecuencia de su entrada en el campo literario, la escritora se ha enfrentado a un fenómeno que podríamos relacionar con el concepto de la paradoja de la alteridad, teorizado por el sociólogo Sayad (2010: 16). El desafío del sociólogo fue desmontar los constructos y determinaciones que se constituyeron respecto al sujeto inmigrante devolviendo a este sujeto una voz y una palabra. Un aspecto fundamental de su estudio en La paradoja de la alteridad (2014) fue el problema de la identidad del inmigrado y las condiciones sociales de su marginalidad, pero también las condiciones sociales que lo determinan y construyen como inmigrante (Gil Araujo, 2010). Sayad resume con una pregunta emblemática — "comment, en effet continuer à être algérien alors qu'on vit hors d'Algérie depuis plusieurs décennies?»² (2014: 116) — perfectamente la paradoja de la alteridad, esto es, una condición paradójica del inmigrante que sigue siendo tal, a pesar de los años que lleve en la sociedad de acogida donde ha trabajado, se ha formado y ha construido una familia.

Si relacionamos esta condición paradójica con el caso de El Hachmi, podemos observar que vive desde los ocho años en Cataluña, escribe y publica sus libros en catalán, aun así, está todavía asociada a la figura del extranjero. El comparatista Armando Gnisci (2003), en su observación sobre los autores de origen inmigrante, formula una pregunta fundamental para nuestro análisis: ¿cualquier escritor que emigra es considerado un autor inmigrante? Si pensamos en los autores latinoamericanos del boom que emigraron a España, y

2. «Cómo podemos seguir siendo argelinos cuando hemos vivido fuera de Argelia durante varias décadas?» (traducción mía). 
en particular a Barcelona, no se ha creado ni en la crítica ni en los medios de comunicación la etiqueta de autor inmigrante. Estos autores pertenecen, según Gnisci (2003), a la misma comunidad interliteraria de los autores españoles, por eso no se ha extranjerizado su figura. ¿’Por qué entonces Najat El Hachmi es una escritora más extranjera que un escritor latinoamericano, aunque ha crecido y vivido toda su vida en Cataluña? Para poder contestar esta pregunta es necesario considerar los distintos grados del ser inmigrante en relación con el lugar de origen (Alcalde, 2011), pero sobre todo comprender que cada campo produce una propia clase de extraño. Retomando la observación de Bauman (1995) de que todas las sociedades producen su propia clase de extraños, en esta investigación es necesario considerar las especificidades de un campo en concreto: el campo literario, donde la figura del extraño y del extranjero se ha producido a partir de sus reglas. Cada sociedad produce su categoría de extraños (Bauman, 1995), pero dentro de estas sociedades el extraño se relaciona de forma diferente dentro de los diferentes campos sociales.

La cuestión de la construcción social de la figura del extraño en el campo literario es diferente a todos los otros campos. La escritora analizada es considerada extraña en el campo cultural local, aunque creció en Cataluña y escribe en catalán y para el público de esta sociedad. La mayoría de las novelas publicadas por ella se relacionan con el tema de la migración. Las críticas y la prensa la asocian con este tema, que también se convierte tanto en una etiqueta como en un recurso estratégico. La componente extranjera en sus novelas se convierte en un factor de la estética y la temática de la novela contemporánea, garantizadora de ventas y consagrada por la crítica (Ricci, 2010). En la tabla número 2,

Tabla 2. Recepción de las novelas de su trilogía en la prensa, en el campo académico/intelectual y los premios recibidos

\begin{tabular}{|c|c|c|c|}
\hline $\begin{array}{l}\text { Novelas relacionadas } \\
\text { con la migración }\end{array}$ & $\begin{array}{l}\text { Crítica literaria en el campo } \\
\text { intelectual/académico }\end{array}$ & Prensa & Premios \\
\hline 2008 - L'últim patriarca & $\begin{array}{l}\text { Ricci (2010). L'últim patriarca, de } \\
\text { Najat El Hachmi, y el forjamiento } \\
\text { de la identidad amazigh-catalana. }\end{array}$ & $\begin{array}{l}\text { Una escritora de origen marroquí } \\
\text { gana el mayor premio de novela } \\
\text { en catalán. Ernest Alós, El Periódi- } \\
\text { co, } 2008 \text {. }\end{array}$ & $\begin{array}{l}\text { Premio Ramon Llull } \\
\text { Premio Ulysse }\end{array}$ \\
\hline 2015 - La filla estrangera & $\begin{array}{l}\text { Darici (2017). Literatura transna- } \\
\text { cional en Cataluña: La filla estran- } \\
\text { gera, de Najat El Hachmi. }\end{array}$ & $\begin{array}{l}\text { «La escritora marroquí Najat El } \\
\text { Hachmi ha sido la ganadora del } \\
35 .^{\circ} \text { premio BBVA Sant Joan de } \\
\text { literatura catalana». Redacción, } \\
\text { La Vanguardia, 2015. }\end{array}$ & $\begin{array}{l}\text { Premio Sant Joan } \\
\text { Premio Ciutat de Barcelona }\end{array}$ \\
\hline 2018 - Mare de llet i mel & $\begin{array}{l}\text { Faszer-McMahon (2020). Women's } \\
\text { Voices from the Maghreb: Trans- } \\
\text { national Feminism in Najat El } \\
\text { Hachmi's Mare de Ilet i mel (2018) } \\
\text { and Lamiae El Amrani's Poesía } \\
\text { femenina y sociedad. }\end{array}$ & $\begin{array}{l}\text { La marroquí Najat El Hachmi } \\
\text { narra en Madre de leche y mie/ la } \\
\text { experiencia como inmigrante de } \\
\text { una mujer, en un relato no exento } \\
\text { de notas melodramáticas y moral- } \\
\text { izantes. Ana Rodríguez Fischer, El } \\
\text { País, } 2015 \text {. }\end{array}$ & \\
\hline
\end{tabular}

Fuente: elaboración propia 
se muestra cómo ha variado la recepción de las novelas de su trilogía en la prensa y en la crítica literaria, además de los premios recibidos.

En referencia a la recepción de sus novelas en la prensa española, podemos observar que la escritora, en los catorce años de trayectoria literaria, desde la primera hasta la última novela sigue siendo considerada una autora de origen extranjero. Como subrayan Blanes y Gibert (2009), en una investigación enfocada al análisis del discurso sobre media y migración en Cataluña, llama la atención la discrepancia entre las entrevistas que hace la prensa a El Hachmi sobre su origen inmigrante, aunque ella sigue afirmando que no se percibe así. Esta discrepancia está fuertemente conectada con una condición negativamente tipificada en casi todas las esferas de la sociedad catalana, sobre todo en la esfera política, dicho de otra manera, una mujer de origen inmigrante, pero de un colectivo específico: el marroquí. Según Anthias y Lazaridis (2000), hay diferentes factores respecto a la construcción negativamente tipificada del colectivo marroquí en Cataluña. Además de importantes factores históricos, estos autores mencionan el reciente síndrome de la invasión islámica en Europa y del terrorismo islámico, que ha creado un alarmismo racista hacia toda la población inmigrante de cultura musulmana. Estas consideraciones ayudan a entender por qué la connotación de El Hachmi con su cultura de origen tiene una raigambre tan marcada. Por ejemplo, es indicativo mencionar los siguientes casos emblemáticos. El País, en un artículo de Ana Rodríguez Fisher del 19 de marzo de 2018, en el suplemento cultural Babelia, presenta su última novela, Madre de leche y miel, de esta forma: «La experiencia como inmigrante de una mujer». Y define El Hachmi como escritora marroquí, no catalana ni tampoco española. Najat El Hachmi publica La filla estrangera en 2015 y, el día después de recibir por esta obra el premio Sant Joan, el periódico La Vanguardia, en un artículo del 17 de junio de 2015, en la sección Libros, subraya que El Hachmi es una escritora marroquí y no catalana: «La escritora marroquí Najat El Hachmi ha sido la ganadora del $35 .^{\circ}$ premio BBVA Sant Joan de literatura catalana». Poco ha cambiado desde 2008, cuando los títulos de los artículos de algunos importantes periódicos, después del otorgamiento del célebre premio literario Ramon Llull, tal y como subrayan Blanes y Gibert (2009: 19), no olvidan el origen de la escritora. Por ejemplo, La Vanguardia utiliza este titular: «Un Llull con acento marroquí». Y El Periódico: «Una inmigrante marroquí gana el mayor premio de novela en la catalán».

Como se analiza anteriormente, la paradoja de la alteridad (Sayad, 2014) a la que se enfrenta la autora está relacionada con el hecho de que, aunque creció y se escolarizó en Cataluña y escribe en catalán, es considerada extranjera. También se relaciona con los procesos de negociación del ser inmigrante como pasaporte necesario para acceder al campo literario. Si por un lado la prensa la asocia únicamente a su origen inmigrante, la crítica literaria da un valor a la negociación que ella hace de su alteridad, aspecto que se revela necesario para ser reconocida y aceptada. La experiencia migratoria caracteriza casi todas sus obras, tanto la primera autobiográfica, Jo també sóc catalana (2004), como las novelas de su trilogía: L'últim patriarca, que fue distinguida con el premio 
Ramon Llull en 2008, La filla estrangera (2015), premio Sant Joan, y Mare de llet $i$ mel (2018). Por otro lado, es significativo que la única novela que no se relaciona con la migración, La caçadora de cossos (2011), es la que ha tenido menos éxito, tanto de ventas como de críticas (Ricci, 2014). La experiencia migratoria le ofrece una característica peculiar que responde a un proceso de consagración. Por ejemplo, el filólogo Cristian Ricci (2010) afirma que los procesos de hibridación que nacen como consecuencia de la experiencia migratoria se trasforman en un hecho estéticamente placentero.

El otorgamiento del Llull es el símbolo de que, por lo menos en los ámbitos intelectuales, lo híbrido ha dejado de ser estigma en Cataluña/España/Europa para convertirse en un hecho éticamente digno de novelar, estéticamente placentero, subversivo y garantizador de ventas. (Ricci, 2010: 73)

En referencia a esto, el próximo apartado trata de la relación de estas obras con un conocimiento nacido a partir de la migración, algo bastante diferente de una novela que únicamente habla de migración.

\section{El poder de la escritura: diferencias entre la posición real de la autora y la posición imaginaria de los personajes}

El Hachmi trasforma la migración en un acto cultural porque vivió esta experiencia y es de origen marroquí, pero también porque se relaciona con un grupo de escritores con la misma trayectoria social que en el campo literario transnacional han luchado para posicionarse y convertir un aparente estigma social en un recurso literario. La escritora aprovecha esta experiencia tanto con el plurilingüismo como con la cultura oral. La filóloga Bueno Alonso (2010: 13), en un estudio sobre L'últim patriarca, valora la importancia del plurilingüismo en esta novela para desterritorializar la escritura posicionando a la autora en un lugar mundial y múltiple. La segunda obra de la trilogía, La filla estrangera, mantiene la misma temática: la historia de una reagrupación familiar narrada por una joven de origen inmigrante. La apuesta por la alteridad se enlaza otra vez a un texto plurilingüe. Darici (2017) analiza la obra de El Hachmi dentro de un contexto de autoras migrantes más amplio y comparativo y las valora como «figuras que se adhieren a una opción lingüística translingüe por el hecho de escribir en catalán y mantienen una subjetividad híbrida, debido a su nacimiento fuera de España». En su última novela, Mare de llet $i$ mel, El Hachmi sigue representando el contexto social de las migraciones contemporáneas, pero esta vez desde el punto de vista de una madre, Fátima: la voz narrativa y protagonista de la novela. Como en las novelas anteriores, la escritora desplaza la historia del Rif marroquí a Catalunya, donde Fátima, ya adulta, casada y madre, emigra con su hija. El plurilingüismo también está presente, y mantiene aquel hilo de representación simbólica de la alteridad. Según Faszer-McMahon (2020: 9), El Hachmi introduce en esta obra una voz feminista transnacional para representar el mundo oral de su comunidad de origen, fuertemente enla- 
zado con una cultura analfabeta y femenina. En las tres novelas analizadas, las protagonistas representan diferentes marginalidades relacionadas con la mujer inmigrante en un determinado espacio social imaginado. Estas marginalidades están asociadas tanto a una condición negativamente tipificada de este colectivo como a una marginalidad de género.

En L'últim patriarca (2008), la protagonista, por su condición femenina, no hereda ningún capital social y cultural de su familia, además los capitales que va buscando la distancian de ella. En su segunda novela, La filla estrangera (2015), la protagonista también va en busca de un ascenso social. Gracias a su buen rendimiento escolar, esta búsqueda separa al personaje del hilo que lo une con su madre, lo que crea una dolorosa y contradictoria ruptura con el habitus femenino de su cultura de origen. La distancia y el choque cultural percibido en el espacio social representado están reflejados en las relaciones madre-hija, situación que empeora los conflictos identitarios. En la última obra de la trilogía, Mare de llet $i$ mel (2018), la protagonista representa la doble ausencia del sujeto inmigrante, con todos los condicionantes de género añadidos: una mujer, madre e inmigrante sin recursos culturales y económicos. En la novela, las reproductoras del dominio patriarcal no son únicamente las figuras masculinas, sino todas las mujeres que las rodean. Abajo se menciona una parte significativa de la obra, donde se representa esta dominación por medio de la madre de Fátima, que limita la posición de su hija exclusivamente a la de la esposa: «Filla meva, havia afegit la mare, la cambra d'una dona, el seu lloc en el món, no és la del seu pare, és la del seu marit» (El Hachmi, 2018: 78). Por otro lado, cuando la narradora desplaza el ojo del lector a la sociedad de llegada, presenta a una mujer sin recursos culturales para hacer frente a los cambios generacionales de su hija, que se enfrenta a las fuerzas asimiladoras de la escuela: "A hores d'ara ja és una completa estrangera. Jo crec que no li agrada la mare que li ha tocat, que preferiria tenir-ne una de cristiana, que llegís, amb qui pogués parlar dels llibres que a ella li interessen» (El Hachmi, 2018: 323).

El Hachmi, aunque se relaciona con las tres protagonistas de su trilogía, por sus obvias proyecciones de corte autobiográfico, al ser ella misma una mujer de origen inmigrante, se disocia de estas representaciones para sacar partido de una alteridad representada mediante la escritura que le da el poder de separarse de sus personajes. Bourdieu (1995: 52), en el análisis de La educación sentimental, observa las posiciones del protagonista, Frédéric, y la estructura del espacio social en la que su propio autor está situado. La profundidad sociológica de este análisis reconoce las diferencias entre el protagonista y Flaubert. Frédéric encarna al joven escritor que, en el interior del campo social, por temor al fracaso, no es capaz de elegir una estrategia clara de posicionamiento, no es capaz de elegir y jugar su carta. Flaubert, al contrario de su personaje, conoce como pocos escritores el campo literario y por eso lo elige como fuente de todas sus motivaciones. Esta elección caracterizará la posición de un escritor que considera el arte mismo como el horizonte último de sus intereses, que Bourdieu analiza como emblema del arte por el arte. «El poder de la escritura» separa Flaubert 
de Frédéric (ibidem). Aunque de forma muy diferente, es posible analizar este aspecto de la separación entre El Hachmi y las protagonistas de sus novelas. Se puede reconocer esta diferencia, ya que, si las protagonistas de su trilogía no son capaces de encontrar una solución para superar sus marginalidades, ella, a través de la escritura, intenta negociar los conocimientos que la experiencia migratoria le ha ofrecido para trasformar y superar estas marginalidades. La novelista va en la búsqueda de una doble herencia cultural, que reconoce la cultura oral amazigh que heredó de su madre, aunque lejana del polo dominante, como un elemento importante de su escritura. Después este aspecto se observa en el acto de traducción permanente, cuando la narradora traduce su cultura de origen en el universo lingüístico y cultural de la sociedad de llegada (Vidal Claromonte: 2012).

Esta separación entre la autora y sus personajes, por un lado, nos ayuda desde un punto de vista metodológico a superar el vínculo mecanicista que superpone la biografía de un autor al contexto social representado en sus novelas; por otro, permite enlazar la teoría del campo a la interpretación de la negociación del origen inmigrante en el contexto literario catalán, que es el segundo objetivo de este estudio. En este aspecto, es necesario considerar que escribir una novela es un acto cultural que permite, a partir de la publicación y circulación, acceder al campo literario. Estas características desvelan, en referencia a la teoría del campo de Bourdieu (1995), que a su vez hacía referencia a Weber, la importancia de lo simbólico en la práctica artístico-cultural (Boschetti, 2003). El Hachmi se distancia de los personajes subalternos que describe e imagina para situarse entre diferentes capitales culturales en un campo concreto: trasforma su otredad literaria en una cualidad consagrada por la crítica literaria. Esta crítica literaria, con un enfoque cultural y poscolonial (Ricci, 2010; Bueno Alonso; 2010) o con un enfoque comparativo y trasnacional (Darici, 2017; Faszer-McMahon, 2020), ha subrayado la importancia que ha tenido la intromisión de una obra híbrida y plurilingüe, fruto de la experiencia migratoria de la escritora en la literatura catalana. El premio Ramon Llull y el Prix Ulysse, por su primera novela, y el premio Sant Joan y el Ciutat de Barcelona, por la segunda, son otros ejemplos significativos de este reconocimiento artístico. Lo que caracteriza Najat El Hachmi es su completa consciencia de que la componente extranjera de su escritura es un valor, por esta razón, su proceso de negociación consiste en demostrar que este componente es parte de la literatura. La autora es consciente tanto de las resistencias que limitan su posición en el campo literario como de las oportunidades relacionadas con su unicidad y particularidad.

Lo que te empuja a escribir es lo que realmente te hace único, es la particularidad que tú tienes, yo sigo con la necesidad de escribir sobre temas relacionados con este contexto. Este contexto social (la migración) me sigue interesando, junto con otras cuestiones, la condición de la mujer, la sexualidad. Yo creo que también el compromiso está en no renunciar, también hay presiones para que te normalices: «Deja ya estos temas y te dedicas a otras cosas, y sé una escritora normal». (Entrevista realizada para esta investigación) 
En la entrevista realizada para esta investigación, la escritora define la presión que la empuja a escribir como mujer de origen inmigrante como positiva, que se enfrenta a una cierta resistencia al extraño. Comenzando desde su toma de posiciones, se pone de manifiesto la necesidad de encontrar la carta ganadora que su habitus con relación al campo le puede ofrecer, la única carta que le permite ser singular en el contexto local, pero fácilmente reconocible en el contexto transnacional. Su lucha para acceder como autora inmigrante está relacionada primero con el contexto local, donde necesita determinadas fuerzas para promocionarse como una de las primeras mujeres inmigrantes. En otras palabras, quiere introducir una realidad ya consagrada en otros contextos (Estados Unidos, Francia, Italia, Inglaterra) en uno más periférico como el catalán. Se podrían citar numerosos ejemplos que, a partir de los años setenta, han caracterizado los grandes cambios globales del mundo literario introduciendo y consagrando la voz de la mujer de origen inmigrante, como los casos de Anzaldua, Cisneros, Monica Ali y Chimamanda Adiche. Todos estos cambios han creado un espacio que El Hachmi ha sabido aprovechar con relación a un campo periférico y singular como el de la literatura catalana (Bellinzis, 2019).

En esta sesión se ha observado cómo la estrategia de posicionamiento de la autora depende de diferentes factores: el género, la clase social, el origen étnico y la generación. En este sentido, la estrecha relación que tiene la autora con la migración como experiencia simbólica, que se refracta en la temática y forma de escribir, no puede ser explicada solo por sus condicionantes sociales. Esta consideración sería reduccionista en el momento en que descuida la complejidad del universo literario. La trayectoria literaria de Najat El Hachmi no se puede observar únicamente por los condicionamientos asociados a un determinado tipo de condición, sino sobre todo a partir de cómo la escritora se posiciona respecto a múltiples condicionamientos dentro del campo literario. Ser mujer, de origen inmigrante, de la generación 1.5, de clase trabajadora y de cultura marroquí constituye un conjunto de disposiciones interiorizadas que informan las percepciones de este sujeto. El habitus de esta escritora no puede, por lo tanto, referirse a un concepto de habitus homogéneo. Es necesario replantear el concepto bourdieusiano dentro del contexto de las migraciones, donde las orientaciones no están enraizadas a una sola condición, sino a múltiples condiciones. Además, el habitus de El Hachmi se orienta hacia una mirada transnacional: del lugar de origen (lengua y cultura), del lugar de llegada (lengua y literatura) y de un contexto transnacional (plurilingüismo y literatura mundial). Por estas razones, se ha puesto en el centro del análisis la complejidad del sujeto inmigrante y la complejidad de las relaciones simbólicas producidas en el campo literario.

Todas las descripciones citadas hasta ahora han sido un elemento del análisis necesario para observar y comprender las cualidades que importa El Hachmi al campo literario a partir de la propia experiencia con el mundo de la migración. La novelista trae consigo una imaginación transnacional que le permite desarrollar la narración entre dos culturas y dos lugares, una cualidad literaria que junto con el plurilingüismo la posiciona en un espacio híbrido. El lector 
de sus novelas se relaciona con una imaginación que desplaza el ojo europeo a un lugar-otro, a través de una narración que reconstruye el vínculo entre los dos lugares. El enfoque planteado ha observado no solamente las relaciones con su país de origen, relaciones identitarias y culturales, sino que además ha interpretado cómo sus productos literarios son síntomas de estas relaciones. Este último planteamiento ha permitido conectar la segunda pregunta de investigación con la tercera. Por lo tanto, la autora negocia no solo su alteridad, sino también el conocimiento de esta mediante una experiencia que se nutre de relaciones transnacionales.

\section{Conclusiones}

El objetivo principal de este artículo ha sido desde el principio la comprensión del campo literario en el cual esta escritora de origen inmigrante ha adquirido un espacio de autorrepresentación. Este trabajo, siguiendo un enfoque de la sociología de la literatura, ha intentado ofrecer una contribución teórica y metodológica al estudio sobre las producciones literarias de autoras/es inmigrantes por haber contextualizado y desmontado aquella idea sobre las relaciones espontáneas entre lenguas y culturas, analizando las relaciones de poder que atribuyen a estos productos artísticos un valor simbólico, fruto de un proceso de negociación de la alteridad migrante. Este aspecto es lo que ha justificado una aportación sociológica al tema, respecto a los estudios literarios, culturales y poscoloniales. Este planteamiento permite una reflexión sobre las especificidades de un campo concreto observando la migración como una experiencia personal que puede convertirse en un instrumento de negociación de diferentes capitales, culturales y simbólicos.

Esta autora cruza diferentes fronteras de clase, pero también étnico-culturales, e introduce en la literatura catalana cualidades externas y una mezcla simmeliana entre cercanía y lejanía cultural (Simmel, 1977). Como afirma Simmel (1977: 83), «el extranjero comparte determinadas características con la sociedad a la que llega, pero trae consigo cualidades que no proceden ni pueden proceder de esa sociedad». Aunque la autora creció en Cataluña y escribe en catalán, entra en el campo gracias a la negociación de la alteridad y se convierte con el tiempo en el otro cercano de una literatura local. Finalmente, este otro, a pesar de llevar casi toda su vida en el país de acogida, sigue siendo otro, pero ya es el otro cercano, porque tiene una posición reconocible, aceptada y en algunos casos consagrada en el campo donde se posiciona, y la alteridad es una componente de esta aceptación y consagración. El Hachmi se mueve en el campo literario trayendo consigo cualidades externas que se han convertido en una oportunidad de posicionamiento. A partir de este argumento, se puede aseverar como interpretación conclusiva de este estudio que la trayectoria literaria de la autora se relaciona simbólicamente con la migración no exclusivamente por un interés sociopolítico y literario, sino también por una necesidad inherente de las posibilidades del campo, donde ha conectado una realidad local, la literatura catalana, a una realidad transnacional, la migración contemporánea. 


\section{Agradecimientos}

Quiero agradecer a la Dra. Bielsa sus preciosas reflexiones sobre el extraño y a la escritora Najat El Hachmi, la disponibilidad mostrada a lo largo de nuestros encuentros.

\section{Referencias bibliográficas}

Alcalde, R. (2011). «De los outsiders de Norbert Elias y de otros extraños en el campo de la sociología de las migraciones». Papers, 96 (2), 375-387. <https://doi.org/10.5565/rev/papers/v96n2.83>

ANTHIAS, F. y LAZARIDIS, G. (2000). Gender and Migration in Southern Europe. Women on the Move. Oxford, New York: eds. Berg.

APARICIO, R. y TORNOS, A. (2006). Hijos de inmigrantes que se hacen adultos: marroquies, dominicanos, peruanos. Madrid: Ministerio de Asuntos Sociales.

BAUMAN, Z. (1995). «Making and unmaking of Strangers». Thesis Eleven, 43 (1), 1-16. <https://doi.org/10.1177/0725513602070001005>

BellinZIS, F. (2019). «The Migrant Female Writer, Originally from Muslim Country in the Literary Field: A Sociological Approach». En: TiBI, Ali y RafiK KHaliL, Rania M. (eds). Culture, Literature and Migration. Londres: Transnational Press London.

Benvenuti, G. y Ceserani, R. (2012). La letteratura nell'età globale. Bolonia: Il Mulino.

BIELSA, E. (2016). Cosmopolitanism and Translation. Investigations into the Experience of the Foreign. Londres y Nueva York: Routledge.

BLANES, L. y GIBERT, B. de et al. (2009). "Elles i nosaltres». Una aproximació al discurs dels mitjans de comunicació catalans sobre les dones immigrades. Barcelona: Acsur Las Segovias.

BoschetTI, A. (2003). La rivoluzione simbolica di Pierre Bourdieu. Venecia: Marsilio Editore.

- (2012). «How Field Theory Can Contribute to Knowledge of World Literary Space». Paragraph, 35 (1), 10-29. $<$ https://doi.org/10.3366/para.2012.0039>

Bourdieu, P. (1979). «Los tres estados del capital cultural». Sociológica, 2 (5). UAM. México DF: Azcapotzalco.

- (1988). Language and symbolic power. Cambridge: Polity Press.

- (1993). The Field of Cultural Production: Essays on Art and Literature. Cambridge: Polity Press.

- (1995). Las reglas del arte. Génesis y estructura del campo literario. Barcelona: Anagrama.

Brouillette, S. (2007). Postcolonial Writers in the Global Literary Marketplace. Londres: Palgrave Macmillan.

Bryman, A. (2012). Social research methods. Oxford: Oxford University Press.

Bueno Alonso, J. (2010). "Género, exilio y desterritorialidad en L'últim patriarca de Najat El Hachmi». En: MiampiKA, Landry-Wilfrid y Arroyo, Patricia (ed.). De Guinea Ecuatorial a las literaturas hispanoafricanas. Madrid: Verbum,.

Casanova, P. (2001). La república mundial de las letras. Barcelona: Anagrama.

CAZENAVE, O. (2003). Afrique sur Seine. Une nouvelle génération de romancier africains à Paris. París: L'Harmattan. 
Cronin, M. (1998). Translation and Identity. Londres y Nueva York: Routledge.

Damrosch, D. (2003). What is World Literature? Princeton: University Press.

DARICI, K. (2017). «Literatura transnacional en Cataluña: La filla estrangera de Najat El Hachmi». Diablotexto Digital, 2, 107-136. <https://doi.org/10.7203/diablotexto.2.10139>

El Hachmi, N. (2004). Jo també sóc catalana. Barcelona: Columna.

- (2008). L'últim patriarca. Barcelona: Planeta.

- (2011). La caçadora de cossos. Barcelona: Planeta.

- (2015). La filla estrangera. Barcelona: Grup 62.

- (2018). Mare de llet $i$ mel. Barcelona: Grup 62.

FASZER-MCMAHON, D. (2020). «Women's Voices from the Maghreb: Transnational Feminism in Najat El Hachmi's Mare de llet i mel (2018) and Lamiae El Amrani's Poesía femenina y sociedad». Transmodernity. Spring Issue, 2020. $<$ https://doi.org/10.5070/T494048546>

Gil Araujo, S. (2010). "Abdelmalek Sayad. Una sociología (de las migraciones) para la resistencia». Empiria, Revista de Metodología en Ciencias Sociales, 19, 235-249.

GNISCI, A. (1998). Creoli meticci migranti clandestini e ribelli. Roma: Meltemi Editore.

- (2003). Creolizzare l'Europa: letteratura e migrazione. Roma: Meltemi Editore.

Goldmann, L. (1967). Para una sociología de la novela. Madrid: Ciencia Nueva.

GUARNIZO, L. (1997). "The emergence of a transnational social formation and the mirage of return migration among Dominican transmigrants». Identities, 4 (2), 281-322. <https://doi.org/10.1080/1070289X.1997.9962591>

HeInCH, N. (2000). Être écrivain. Création et identité. París: Ed. La Découverte.

INGENSCHAY, D. (2011). «Migraciones e identidades en L'últim patriarca de Najat El Hachmi». Iberoromania, 71-72 (1), 57-70. <https://doi.org/10.1515/iber.2010.006>

KarrouCH, L. (2004). De Nador a Vic. Barcelona: Columna.

LaHiRe, B. (2010). Franz Kafka. Éléments pour une théorie de la création littéraire. París: La Découverte.

- (2011). Ce qu'ils vivent, ce qu'ils écrivent. Mises en scène littéraires du social et expériences socialisatrices des écrivains. París: Éditions des Archives Contemporaines.

LAMrI, T. (2002). «Il pellegrinaggio della voce» En: AmmEndolA, S. Parole di sabbia. Savona: Edizioni Il Grappolo.

LUKÁCS, G. (1966) Sociología de la literatura. Madrid, Península.

Marco, J. (2010). "Una aproximació a l'habitus de Carles Capdevila, traductor i home de lletres». Quaderns: Revista de Traducció, 17, 83-104.

Meizoz, J. (2010). «L'individuel et le social» En: BoschetTi, Anna (eds). L'espace culturel transnational. París: Nouveau Monde Éditions.

MengOzZI, C. (2013). Narrazioni contese. Vent'anni di scritture italiane della migrazione. Roma: Carrocci.

Moretti, F. (1996). The Modern Epic: The World-System from Goethe to Garcia Márquez. Londres, Nueva York: Verso.

Rama, A. (1985). Transculturación narrativa en América Latina. Ciudad de México: Siglo XXI.

Ricci, C. H. (2010). «L'últim patriarca de Najat El Hachmi y el forjamiento de la identidad amazigh-catalana». Journal of Spanish Cultural Studies, 11.1, 71-91. <https://doi.org/10.1080/14636201003787535>

- (2014). ¡Hay moros en la costa! Literatura marroqui fronteriza en castellano y catalán. Madrid: Ediciones de Iberoamericana. 
SAID, E. (1975). Beginnings: Intention and Method. Nueva York: Columbia University Press.

- (1979). Orientalism. Nueva York: Vintage Books.

SAPIRO, G. (2009). Les contradictions de la globalisation éditoriale. París: Éditions Nouveau Monde.

- (2013). «Le champ est-il national: La théorie de la différenciation sociale au prisme de l'histoire globale». Actes de la Recherche en Sciences Sociales, 200 (5), 70-85. <https://doi.org/10.3917/arss.200.0070>

- (2014). La sociologie de la littérature. París: Ed. La Découverte.

SAYAD, A. (2010). La doble ausencia. De las ilusiones del emigrado a los padecimientos del inmigrado. Barcelona: Anthropos.

- (2014). L'immigration ou les paradoxes de l'altérité. 3. La fabrication des identités culturelles. París: Éditions Raisons d'Agir.

Simmel, G. (1977). Sociologia: Estudios sobre las formas de socialización. Tomo 2. Madrid: Revista de Occidente.

SpIvaK, G. (1990). The Post-Colonial Critic: Interviews, Strategies, Dialogues. Nueva York: Routledge.

TURNATURI, G. (2003). Immaginazione sociologica e immaginazione letteraria. Roma: Laterza.

VerTOVEC, S. (2004). «Migration and other Modes of Transnationalism: Towards Conceptual Cross-Fertilization». International Migration Review, 37 (3), 641-665. $<$ https://doi.org/10.1111/j.1747-7379.2003.tb00153.x>

Vidal Claromonte, A. (2012). «Jo també sóc catalana: Najat El Hachmi, una vida traducida». Quaderns: Revista de Traducció, 19, 237-250.

Wimmer, A. y Glick SCHILler, N. (2002). «Methodological nationalism and beyond: nation state building, migration and the social sciences». Global Networks, 2 (4), 301-334. <https://doi.org/10.1111/1471-0374.00043>

YILDIZ, Y. (2012). Beyond the Mother Tongue. The postmonolingual condition. Nueva York: Fordham University Press. 
